Research Article

\title{
Validation Study of Mobile Lab for Clinical Biochemistry in Rural Population of Delhi
}

\author{
Jugal Kishore', Nalini Tripathi', Neeta Kumar ${ }^{3}$, Amit Bhatnagar ${ }^{4}$, Lal Chandra ${ }^{5}$
}

${ }^{1}$ Director Professor \& Head, ${ }^{2}$ Scientist-C, ICMR Project, Department of Community Medicine, VMMC \& Safdarjung Hospital, New Delhi.

${ }^{3}$ Scientist-E, Division of Innovation Translation and Reproductive Biology Maternal Child Health, ICMR.

${ }^{4} \mathrm{MD}$, Accuster technologies Pvt. Ltd. Gurugram, Haryana.

${ }^{5}$ Professor, Department of Biochemistry, MAMC, New Delhi.

DOI: https://doi.org/10.24321/2394.6539.201902

\section{I $\quad \mathbf{N} \quad \mathbf{F} \quad \mathbf{O}$}

\section{Corresponding Author:}

Nalini Tripathi, Division of Innovation Translation and Reproductive Biology Maternal Child Health, ICMR.

E-mail Id:

neha.nalini@gmail.com

\section{Orcid Id:}

https://orcid.org/0000-0002-1159-8672

How to cite this article:

Kishore J, Tripathi N, Kumar N, Bhatnagar A, Chandra L. Validation Study of Mobile Lab for Clinical Biochemistry in Rural Population of Delhi. J Adv Res Med Sci Tech 2019; 6(1\&2): 7-14.

Date of Submission: 2019-12-20

Date of Acceptance: 2020-01-18

\section{$\begin{array}{llllllll}\mathbf{A} & \mathbf{B} & \mathbf{S} & \mathbf{T} & \mathbf{R} & \mathbf{A} & \mathbf{C} & \mathbf{T}\end{array}$}

Introduction: Prevention of risk factors and management of Noncommunicable diseases are possible and their complications can be prevented by early diagnosis but due to unavailability of diagnostic facilities at cheaper rates most of the patients remains undiagnosed in rural setting. However, validity of such device is important before its use in these areas.

Objective: To generate validation data using Mobile Lab (Labike \& Suitcase model) to check its sensitivity and specificity against gold standard methods.

Material and Methods: A cross-sectional comparative study was conducted among 824 participants, aged 18 years and above in rural catchment area of the department. Pre-tested questionnaires were used. For the Biochemical investigations blood samples were collected and were divided into two parts for each parameter. One part of the blood sample was kept for the Mobile lab investigations whereas another part was transported to a tertiary care teaching institution for the estimation. The comparison of the results was done through the CLIP range of total Allowable error to define the acceptable limits. Bland and Altman plots are also used for describing the agreement between two quantitative measurements.

Result: Biochemical estimation were done for total 18 parameters in which Uric Acid, Creatinine (-9.39\%), Total Bilirubin (19.8\%) SGOT (16.3\%), SGPT (-17.6\%), ALP (-14.7\%), HDL (16.7\%), Triglycerides (-16.8\%) and Phosphorus $(-10.3 \%)$ were within allowable limits.

Conclusion: Mobile lab is acceptable for most of the any biochemical parameters and can be used as the point of care device for diagnostic purpose with certain correction required for the measurement of Electrolyte panel and Direct Bilirubin test.

Keywords: Mobile Lab, Labike, Diagnostic Lab, Biochemical Parameters, CLIP 


\section{Introduction}

Validation is important and used during accreditation of clinical laboratories and certification, using common standards and practices. Regulatory agencies require that a laboratory validate an instrument before it is put into use for patient testing. ${ }^{1,2}$ From a medical perspective, the value of an automated clinical chemistry analyser is to provide physicians and other health care providers with valid and reliable clinical data for patient management. All results must be statistically and medically comparable on any clinical chemistry analyser. ${ }^{3,4,5}$ Before reporting patient test results, the laboratory needs to demonstrate the accuracy and precision of its analyser. ${ }^{7}$

Laboratory results influence $70 \%-75 \%$ of medical diagnosis hence quality of laboratory service directly affects the quality of health care. Laboratory results must be as accurate as possible and laboratory operations must be reliable, and reporting must be timely in order to be useful in a clinical or public health setting. If inaccurate results are provided, the consequences can be very serious for example, there may be unnecessary treatment, treatment complications, failure to provide the proper treatment, delay in correct diagnosis and additional and unnecessary diagnostic testing. ${ }^{8}$

Several concerns related to Indian health care and its diagnostic services have been raised. In rural areas Laboratory diagnostic services are very limited. Patients need to go far for the health check-up and the diagnostic services. Keeping these facts in mind the Mobile lab was invented for the remote and rural areas for preventive care facilities before the disease get worse. This one-time study was done to find out the validity and reliability and to test the capacity of the semiautomatic biochemistry analyser (Mobile lab) as a point of care device used for the diagnostic services in the remote and rural areas. The present study involves a comparison of test values between two machines i.e.) a fully automated machine which we refer to as the Gold Standard and ii) a semi-automated Mobile lab i.e. test machine. The aim of this study is also to check the operational feasibility of the Mobile lab for providing effective healthcare diagnosis in remote and rural areas.

\section{Materials and Methods}

It was a cross-sectional comparative validation study. A total number of 824 participants aged 18 years and above attending the OPD were included in the study. The precision with which we can estimate Standard Deviation $\left(s_{w}\right)$ depends on both the number of subjects, $n$ and the number of observations per subject, $m$. The width of the $95 \%$ confidence interval for the population within-subject standard deviation is:

$$
1.96 \frac{s_{w}}{\sqrt{2 n(m-1)}}
$$

on either side of the estimate. If we can take only two measurements (Two tests) per subject, and if $10 \%$ on either side of estimate, for example, we have $m=2$ giving us:

$$
\frac{1.96}{\sqrt{2 n \times(2-1)}}=0.10
$$

hence

$$
\frac{1.96^{2}}{2 \times 0.10^{2} \times 1}=192
$$

For accuracy we required a sample size of 192 and if further reduce the confidence interval of $5 \%$ then sample size would be 768 . However, in this study a total of 824 subjects were included.

The study was conducted a Rural Health Training Center, Nazafgarh under Vardhman Mahavir Medical College (VMMC) \& Safdarjung Hospital, New Delhi which was a study site and Department of Biochemistry, Maulana Azad Medical College \& Lok Nayak Jai Prakash Hospital, New Delhi as the Gold standard laboratory. A predesigned, pretested, semistructured questionnaire (in Hindi) was used containing items to access identification data and socio-economic status like, age, sex, religion, marital status, monthly income, family members, education details, professional deals etc. besides risks factors like family history, smoking etc.

For the biochemical estimation, a maximum of $10 \mathrm{ml}$ blood was collected through venupuncture method to test LFT (Albumin, Total Bilirubin, Direct Bilirubin, SGPT, SGOT, and ALP), KFT (Urea, Uric Acid and Creatinine), Glucose (R), Lipid Profile (HDL, Triglyceride and Cholesterol), Electrolytes (Calcium, Sodium, Potassium) and $\mathrm{Hb}$ (Haemoglobin) parameters. These tests were measured using control samples (Bio-Rad) of both levels normal \& pathological (L1 \& L2) in two analyser's i.e. automated (Gold standard) and semi-automated (Mobile lab).

The blood samples were divided into two parts. One part of the sample was sent to THE standard lab of Lok Nayak Hospital, New Delhi and second part was analysed through the Mobile lab (Suitcase and Labike model) at the RHTC Nazafgarh, Delhi itself.

In this study we have carried out a validation procedure for a new machine i.e., a Mobile Lab which is a portable laboratory with a semi-automated analyser and can be used in a suitcase (Suitcase model) or on Labike (Labike model). It is the first product in medical diagnostic field where conception of technology to final product is designed keeping in mind the needs and suitability 
of India. It is a compact lab in a suitcase with Solar and Battery power backup. It has been designed to perform 23 vital biochemistry tests for assessing the functions of Kidney, Liver, Heart and Diabetes accurately, cost-effectively and timely. It includes different components as, blood analyser, centrifuge, micropipettes, incubator, Laptop with Patient Data Management Software and consumables. Key advantage of Mobile Lab is in its design i.e. rugged Analyser, portability and cost effectiveness. Whereas, the Labike model is a mini mobile lab which is installed on a bike and could test additional parameters like, Serology, Hematology, ECG,BP, BMI etc. It has a proper place for reagents and other consumables. It is so rugged that it can be easily carried to the far flung and remotest locations.

\section{Statistical Analysis}

The data entered in the excel sheet and analysis were done using Comparability Testing where the comparison of test results, between two or more instruments within the same laboratory or laboratories at different sites that may process samples from the same patients, to insure measurements that are similar and can be used interchangeably without causing clinical error. In this type of testing Total Allowable Error (TEa) is one tool that is used. It is a commonly used for quality requirement which is derived from medically important analyte concentrations or clinical decision thresholds. ${ }^{9}$

The analysis were carried out by calculating Total Calculated Observed Error (TEobs) using mean of differences, Coefficient of variation and Bias and comparing the TEobs with Total Allowable Error (TEa) guidelines e.g. as mandated by CLIA (Clinical Laboratory Improvement Amendment). ${ }^{10}$ If calculated total allowable error (TEobs) for all concentrations is less than that which is acceptable (Tea), then the instrument performance is satisfactory and no further assessment for that analyte is required. If calculated TEobs is greater than that which is acceptable (TEa), attempts should be made to identify and correct causes of imprecision (high CV) and inaccuracy (high bias). ${ }^{11}$ As both the methods (Gold standard vs. Mobile lab) are measuring the same blood sample, high correlations are expected.

\section{Specific Calculations Used in the study}

Coefficient of Variation (CV): A measurement of imprecision (random error); mathematically, CV is standard deviation divided by mean or average and expressed as a percentage.

Bias (\%): Total systematic error, which includes constant and proportional bias. Presence of bias may be due to multiple factors. It is difference between the measure results and the concentration of a known standard. Generally used to describe the inaccuracy of a method relative to a comparative method in a method comparison experiment. The term bias has a specific meaning in the statistical t-test and in difference plot analysis, where bias (expressed as analyte units) equals the differences between the paired sample values. Bias may also be expressed as a percentage according to the formula;

[Bias $(\%)=$ mean $_{\text {target }}-$ mean $_{\text {measured }} /$ mean $\left._{\text {target }} \times 100\right]^{2}$

Observed or Calculated Total error (TEobs): The sum of random error (imprecision) and systematic error (bias). TEobs is defined in the guideline as $2 \mathrm{CV}+\mathrm{Bias}(\%)$ or 2SD + Bias (units of the analyte). The calculated TE is specific for a single instrument/ method.

Bland-Altman plots (B \& A Plots): B \&A Plots are also being calculated for evaluation of the degree between two diagnostic tests measuring the same variable, using SPSS software and then rechecked by Medcalc software. ${ }^{12}$ In B \& A Plot, the difference between the measurements (e.g. mean urea mobile lab-mean urea gold standard) on $Y$-axis against the mean of the two measurements [(mean urea mobile lab + mean urea gold standard)/2] on X axis is calculated. The mean of the two measurements represents the "best approximation" of the true value sought i.e. the actual amount of urea in the blood. The difference the measurements reflect the amount of disagreement of each paired measurement and may be either positive or negative. The mean and the Standard Deviation (SDs) of the differences between measurements is then calculated. Finally, the upper and lower limits of the agreement and be calculates as the mean +/-2SDs. If the interval between the upper and the lower limits of agreement is small, it may be concluded that the tests are in close agreement and may be substituted for each other. B\& A plot recommends that $95 \%$ of the data points should lie within $\pm 2 S$ of the mean difference. B\&A plot method only defines the intervals of agreements, it does not say whether those limits are acceptable or not.

\section{Ethical Clearance and Informed Consent}

The study was carried out after approval of VMMC \& SJH Institutional Ethical Committee. The participants were briefed about the purpose of the study and informed consent was obtained prior to the data collection. The Informed Consent Form (ICF) and Participant In formation Sheet were used in local language for the understanding of the participants. The mobile lab has helped in providing free diagnostic services to the participants involved in study for many non-communicable diseases.

\section{Result}

The details analysis with the socio-demographic profile is given below: 
Table I.Socio- demographic characteristics of R.H.T.C, Najafgarh

\begin{tabular}{|c|c|c|c|}
\hline S. No. & Characteristics & Total Number $(\mathrm{N}=824)$ & Percentage (\%) \\
\hline \multirow[t]{3}{*}{1.} & Gender & & \\
\hline & Male & 299 & 36.3 \\
\hline & Female & 525 & 63.7 \\
\hline \multirow[t]{7}{*}{2.} & Age in years & & \\
\hline & $18-25$ & 224 & 27.2 \\
\hline & $25-35$ & 141 & 17.1 \\
\hline & $36-45$ & 173 & 21.0 \\
\hline & $46-55$ & 116 & 14.1 \\
\hline & $56-65$ & 104 & 12.6 \\
\hline & Above 66 & 66 & 8.0 \\
\hline \multirow[t]{5}{*}{3.} & Religion & & \\
\hline & Hindu & 781 & 94.8 \\
\hline & Muslim & 38 & 4.6 \\
\hline & Sikh & 1 & 0.1 \\
\hline & Christian & 4 & 0.5 \\
\hline \multirow[t]{3}{*}{4.} & Education & & \\
\hline & Illiterate & 150 & 18.2 \\
\hline & Literate & 674 & 81.8 \\
\hline \multirow[t]{5}{*}{5.} & Marital status & & \\
\hline & Married & 616 & 74.8 \\
\hline & Unmarried & 198 & 24.0 \\
\hline & Separate & 1 & 0.1 \\
\hline & Widow & 9 & 1.1 \\
\hline \multirow[t]{8}{*}{6.} & Occupation & & \\
\hline & Professional & 19 & 2.3 \\
\hline & Semi-professional & 20 & 2.4 \\
\hline & Clerk/ Shop owner/ Farm & 36 & 4.4 \\
\hline & Skilled & 45 & 5.5 \\
\hline & Semi-skilled & 66 & 8.0 \\
\hline & Unskilled & 76 & 9.2 \\
\hline & Unemployed & 562 & 68.2 \\
\hline \multirow[t]{4}{*}{7.} & Monthly Family Income & & \\
\hline & Rs. $0-5000$ & 501 & 60.8 \\
\hline & Rs. $5001-10000$ & 282 & 34.2 \\
\hline & Rs. $10001-50000$ \& above & 41 & 5.0 \\
\hline
\end{tabular}

Table 1, shows socio demographic characteristics for the Najafgarh site. Males were $36.3 \%$ and females were 63.7 $\%$. Majority of participants were in the age group of 18 25 years i.e $27.2 \%$ followed by $36-45$ years $(21.0 \%)$ and
25-35 years (17.1\%). Majority were Hindus (94.8\%) and literate $81.8 \%$. $74.8 \%$ subjects were married and $68.2 \%$ were unemployed including housewives. The monthly family income of $60.8 \%$ subjects was within Rs.0-5000. 
Table 2, comprises of Gold standard values, Test machine values and their difference with standard Deviation (SD), Coefficient of Variation (CV), Bias (\%), Observed Total Error and Allowable Total error. From this table it can be clearly observed that only 3 out of 18 parameters as; $\operatorname{ALP}(28.6), \mathrm{HDL}(21.2)$ and Triglyceride (25.8) are within range of prescribed Allowable Total Error (TEa) however other parameters are out of limits. If we consider the allowable total error up to the range of $\pm 20 \%$ which is acceptable for most of the biochemical test then out of eighteen parameters, 6 parameters as; Urea (18.5), Albumin (17.7) and Total Protein (14.9.3) would be considered with in allowable limit.

Table 2.RHTC Najafgarh Biochemistry data showing the Observed calculated Total Error (TEobs) vs. Allowable Total Error

$(n=824)$

\begin{tabular}{|c|c|c|c|c|c|c|c|c|}
\hline $\begin{array}{l}\text { S. } \\
\text { No. }\end{array}$ & $\begin{array}{c}\text { Test } \\
\text { Parameter }\end{array}$ & $\begin{array}{c}\text { Gold } \\
\text { standard } \\
\text { (Mean士S.D) }\end{array}$ & $\begin{array}{l}\text { Test Machine } \\
\text { (Mobile lab) } \\
\text { (Mean士S.D) }\end{array}$ & $\begin{array}{l}\text { Difference } \\
\text { of Mean } \\
\text { (Mean } \pm \text { S.D) }\end{array}$ & $\begin{array}{l}\text { Coefficient } \\
\text { of Variation } \\
\text { (CV) } \%\end{array}$ & Bias \% & $\begin{array}{l}\text { Observed } \\
\text { calculated } \\
\text { Total Error } \\
\text { (TEobs) \% }\end{array}$ & $\begin{array}{c}\text { Allowable } \\
\text { Total } \\
\text { Error* } \\
\text { ( } \pm \text { \%) }\end{array}$ \\
\hline 1. & Urea & $27.03 \pm 12.21$ & $29.89 \pm 9.88$ & $-2.86 \pm 11.37$ & -3.97 & -10.58 & -18.53 & \pm 9 \\
\hline 2. & Uric Acid & $4.23 \pm 2.34$ & $4.75 \pm 2.38$ & $-0.51 \pm 2.51$ & -4.83 & -12.27 & -21.9 & \pm 17 \\
\hline 3. & Creatinine & $0.92 \pm 0.58$ & $0.80 \pm 0.38$ & $0.12 \pm 0.57$ & 4.61 & 13.48 & 22.72 & \pm 15 \\
\hline 4. & T. Bilurubin & $0.75 \pm 1.01$ & $0.86 \pm 1.21$ & $-0.10 \pm 0.73$ & -6.97 & -13.90 & -27.86 & \pm 20 \\
\hline 5. & D. Bilurubin & $0.27 \pm 1.40$ & $0.36 \pm 1.81$ & $-0.09 \pm 0.86$ & -9.38 & -33.59 & -52.36 & \pm 20 \\
\hline 6. & Albumin & $3.83 \pm 1.47$ & $3.66 \pm 1.37$ & $0.17 \pm 1.15$ & 6.60 & 4.56 & 17.77 & \pm 10 \\
\hline 7. & SGOT & $37.94 \pm 71.10$ & $45.16 \pm 42.57$ & $-7.21 \pm 43.88$ & -6.07 & -19.02 & -31.18 & \pm 20 \\
\hline 8. & SGPT & $31.74 \pm 26.14$ & $35.98 \pm 38.37$ & $-4.23 \pm 43.43$ & -10.26 & -13.34 & -33.86 & \pm 20 \\
\hline 9. & ALP & $104.68 \pm 45.36$ & $77.85 \pm 36.26$ & $26.82 \pm 40.47$ & 1.50 & 25.62 & 28.64 & \pm 30 \\
\hline 10. & Gluocose (R) & $99.22 \pm 44.68$ & $100.24 \pm 36.84$ & $-1.13 \pm 20.98$ & -18.49 & -1.14 & -38.12 & \pm 10 \\
\hline 11. & T.Protein & $7.49 \pm 3.39$ & $7.05 \pm 3.99$ & $0.44 \pm 2.00$ & 4.52 & 5.91 & 14.96 & \pm 10 \\
\hline 12. & $\mathrm{HDL}$ & $41.57 \pm 13.02$ & $38.80 \pm 21.33$ & $2.76 \pm 20.14$ & 7.28 & 6.65 & 21.22 & \pm 30 \\
\hline 13. & TG & $140 \pm 76.20$ & $108.86 \pm 75.07$ & 1.64 & 22.59 & 25.59 & 25.88 & \pm 25 \\
\hline 14. & T. Cholesterol & $170 \pm 47.73$ & $144 \pm 52.32$ & $26 \pm 36.52$ & 1.42 & 15.14 & 17.98 & \pm 10 \\
\hline 15. & Calcium & $9.17 \pm 6.99$ & $8.75 \pm 3.99$ & $0.42 \pm 5.60$ & 13.24 & 4.60 & 31.10 & \pm 8 \\
\hline 16. & $\begin{array}{c}\text { Sodium in } \\
\mathrm{mmol} / \mathrm{It}\end{array}$ & $137.42 \pm 6.92$ & $129 \pm 39.74$ & $8.19 \pm 40.46$ & 4.93 & 43.26 & 53.13 & \pm 0.4 \\
\hline 17. & $\begin{array}{l}\text { Pottasium } \\
\mathrm{mmol} / \mathrm{It}\end{array}$ & $4.39 \pm 0.63$ & $4.36 \pm 1.05$ & $0.03 \pm 1.10$ & 34.66 & 0.72 & 70.05 & \pm 0.5 \\
\hline 18. & Phosphorus & $3.41 \pm 0.99$ & $4.67 \pm 3.72$ & $-1.25 \pm 3.84$ & -3.06 & -36.81 & -42.93 & $\pm 10-23$ \\
\hline
\end{tabular}

${ }^{*}$ According to ASVCP guidelines 2013.

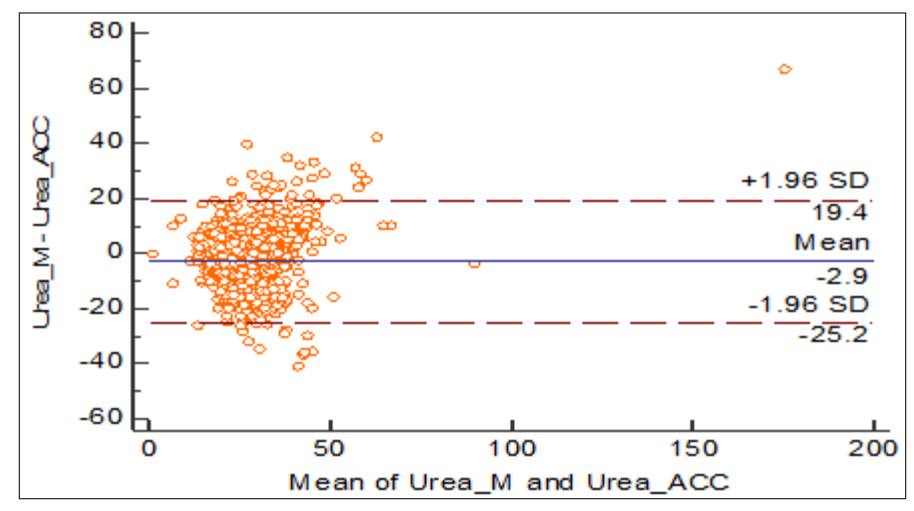

Figure I.Plot of Difference against mean for Urea values 


\section{Bland \& Altman Plot for Najafgarh Data}

Bland \& Altman plot for Urea, Uric Acid, Albumin, Creatinine, Total Bilirubin, SGOT, ALP, Total Protein, HDL, Cholesterol, Triglycerides, Calcium \& Potassium shows that the data points are lying between mean differences (d) and the standard deviation of the differences (s) i.e d-2s and d $+2 \mathrm{~s}$, or more precisely, $95 \%$ of differences are in between $\mathrm{d}-1.96 \mathrm{~s}$ and $d+1.96 \mathrm{~s}$. The results show that two instruments are giving acceptable readings and differences are within limit.

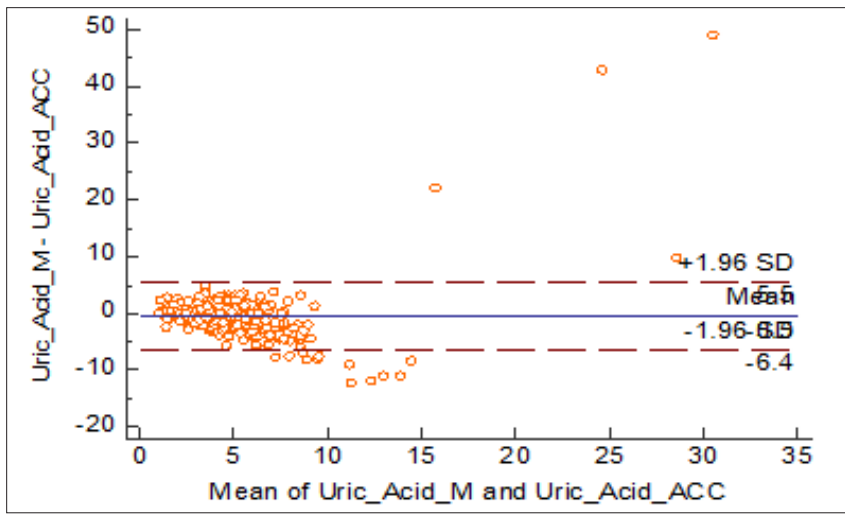

Figure 2.Plot of Difference against mean for Uric acid values

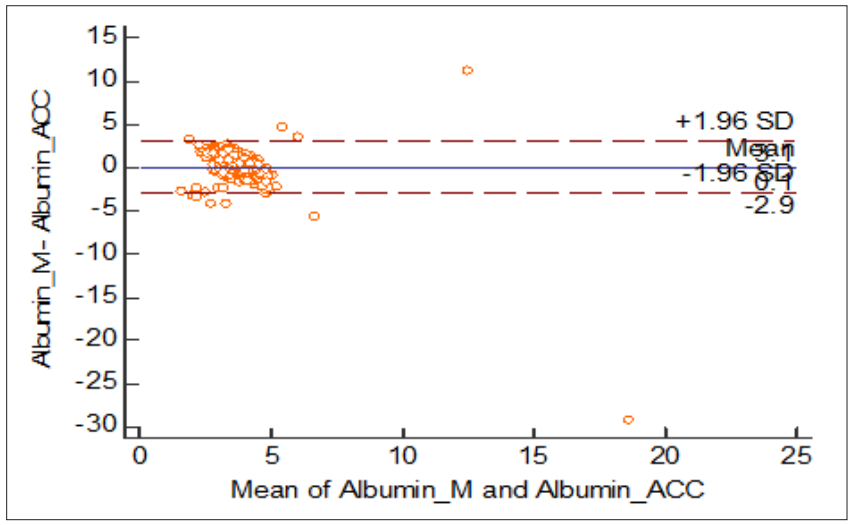

Figure 3.Plot of Difference against mean for Albumin values

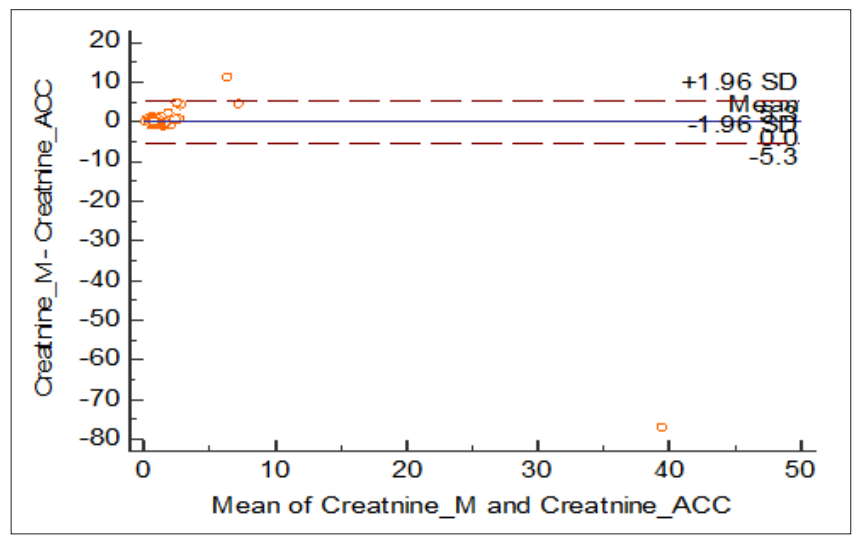

Figure 4.Plot of Difference against mean for Creatnine values

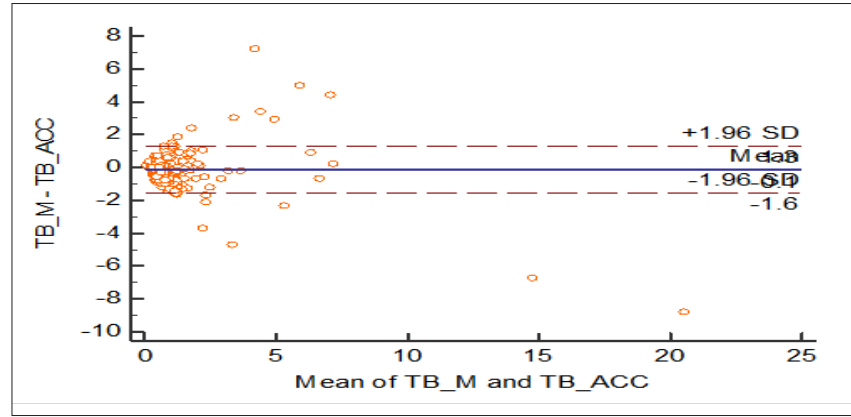

Figure 5.Plot of Difference against mean for Total Bilirubin values

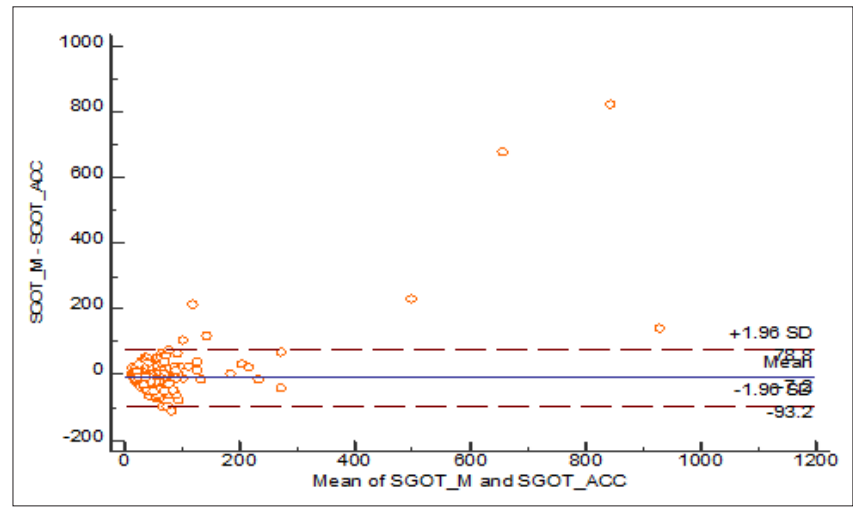

Figure 6.Plot of Difference against mean for SGOT values

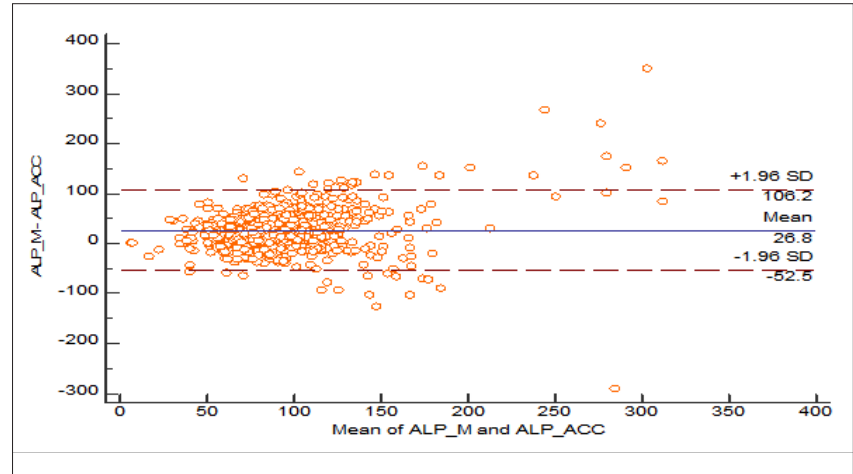

Figure 7.Plot of Difference against mean for ALP values

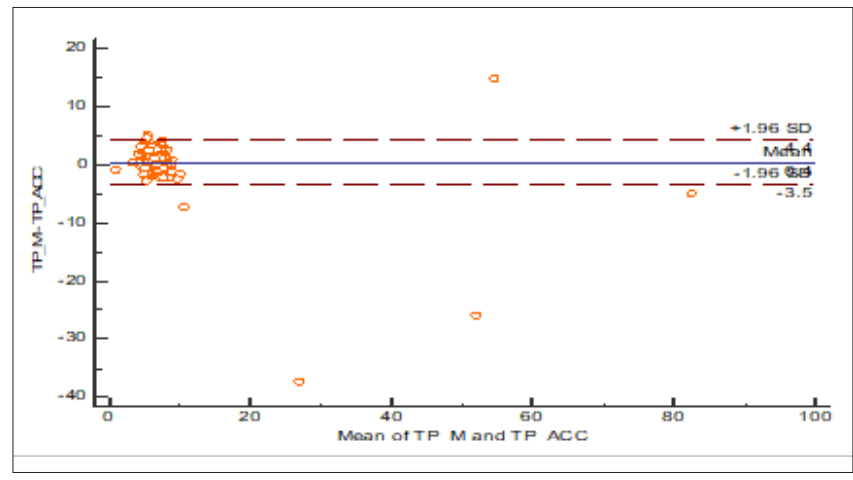

Figure 8.Plot of Difference against mean for Total Protein values 


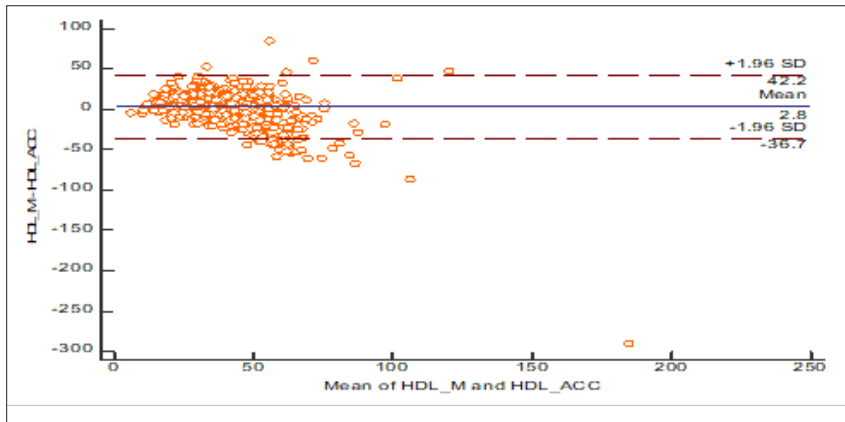

Figure 9.Plot of Difference against mean for HDL values

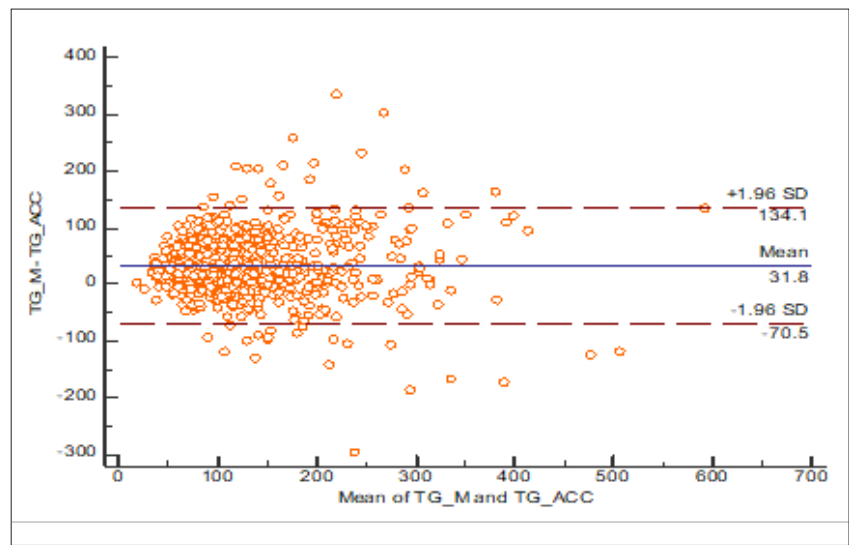

Figure 10.Plot of Difference against mean for Triglyceride values

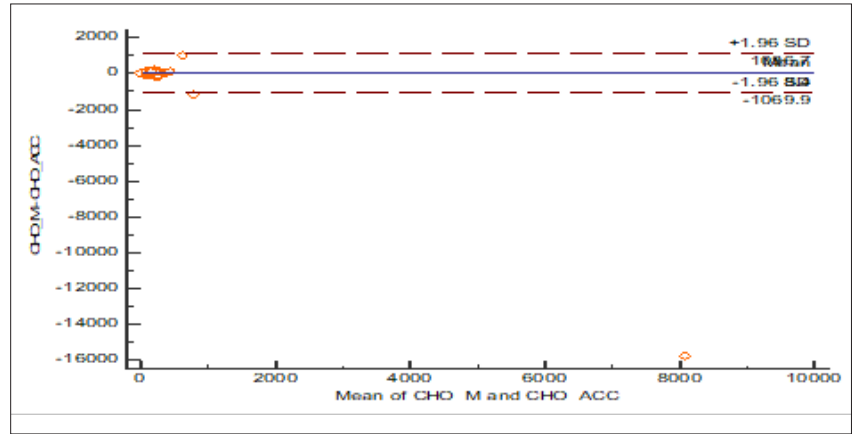

Figure I I.Plot of Difference against mean for Cholesterol values

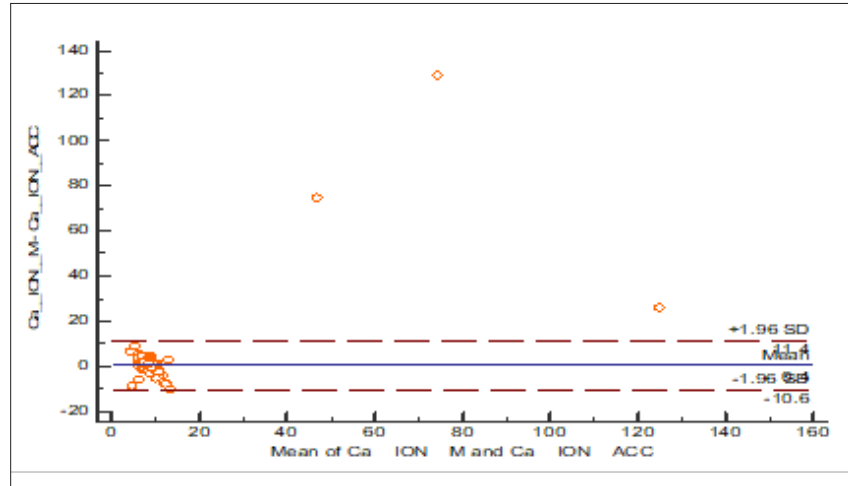

Figure I2.Plot of Difference against mean for Calcium values

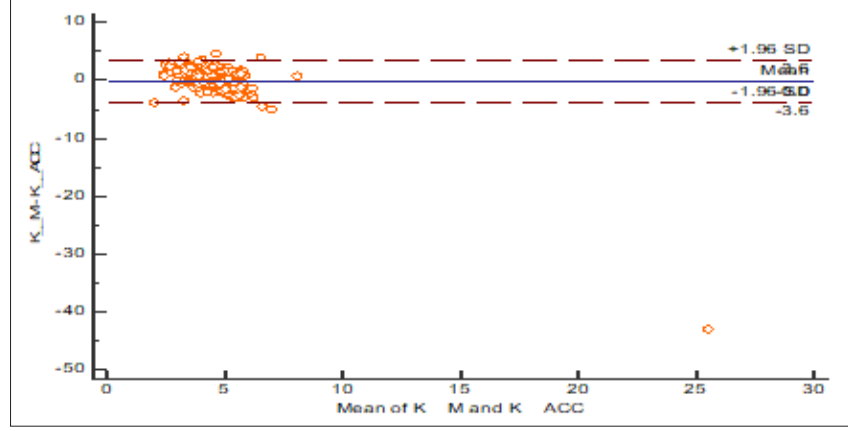

Figure 17.Plot of Difference against mean for Potassium values

However, Bland and Altman plot for Direct Bilirubin, Phosphorus, Sodium, SGPT and Glucose shows proportional error i.e. as the mean of devices increases or decreases, variation in the measurement of devices also increases suggesting inaccurate measurement at lower or higher readings. A negative trend seems to be evident along the graph. Drawing a regression line of the differences could help in detecting a proportional difference.

\section{Discussion}

From the results we can state that the test machine i.e. Mobile lab is acceptable as the point of care device for most of the biochemical parameters and haemoglobin test. It has provided acceptable limits for Uric Acid, Creatinine, Total Bilirubin, SGOT, SGPT, ALP, HDL, TG, Phosphorus and simultaneously it also shows a fair agreement with Urea, Total protein, Glucose, Cholesterol and Calcium. Thus, we can conclude that the mobile lab is good for the abovementioned parameters with some correction required for the measurement of Electrolyte panel and Direct Bilirubin test. However, some of the test parameters were not found acceptable because of various limitations.

Limitations of the Test Machine: Since we know that the accuracy depends upon various factors like room temperature, humidity, reagent condition, machine maintenance, calibration etc., thus change in one factor affects a change in other and so on. There are some limitations for the test instruments and if these are corrected or taken into consideration most of the parameters could come into the prescribed limit of allowable error. The difference in observation observed during the project period could be due to various other factors which are mentioned below:

\section{Manual Error}

- Calibration of the Machine: The calibration is required timely i.e. oncein a week to provide accurate results.

- Quality Check (QC): QC should be run as required for the proper functioning of the machine.

- Pipetting of Sample \& Reagents: This is the most important factor in the procedure. If the pipetting of 
the sample and reagents are not accurate the results would be hampered.

- Incubation Time: There is a fix timing for the incubation of all the parameters, if the incubation time increases or decreases the discrepancy of results would occur.

- Proper Mixing of the Sample \& Reagents: The sample \& reagents should be properly mixed, otherwise exact colour would not appear. This will also cause error in the results.

- Bubble Formation: If there is any bubble in the cuvette, it would also hamper the results. In this case the cuvette should be tapped to remove the bubble.

- $\quad$ Storage of Reagents and Controls: The reagents and controls should be stored properly as prescribed on the bottle label and should be used in working condition. It should not be used beyond the expired date.

\section{Availability of Resources at Project Site}

For the proper functioning of the test machine/instrument, it is very important to provide all the necessary resources like electricity, procurement of reagents \& controls on time, servicing \& maintenance of the machine etc., failing which the test results would hamper and could not be provided in timely and effective manner.

\section{Training of the Staff/ Technicians}

This is the most important aspect for the smooth functioning of the Mobile lab/ Test machine. The staff should be well trained on the instrument and its each and every test parameter. The staff should be trained on the components of the mobile lab and they should be able to fix the trouble shooting in case there is any error in the machine.

In the present study it is observed that the mobile lab can be used as point of care device if proper management and the training of the staff is done. It can be a boon to the rural population where the proper diagnostics and pre-screening facilities are not available.

\section{Acknowledgment}

We thank Indian Council of Medical Research (ICMR) for providing financial support 5/3/8/241/2014-RHN E-office 71871.

\section{Conflict of Interest: None}

\section{References}

1. Clinical and Laboratory Standards Institute (CLSI), Evaluation of Precision Performance of Quantitative Measurement Methods, (3rd Ed.) (CLSI document EP5-A2, ISBN 1-56238-542-9, Pennsylvania, USA, 2004).

2. Hauck WW, Koch W, Abernethy D, Williams RL. Making sense of trueness, precision, accuracy, and uncertainty. Pharmacopoeial Forum 2008; 34(3): 838-842. Available from: https://jhu.pure.elsevier.com/en/publications/ making-sense-of-trueness-precision-accuracy-anduncertainty-3 [Google Scholar].

3. Clinical and Laboratory Standards Institute (CLSI), User verification of performance for precision and trueness (2nd Ed.) (CLSI document EP15-A2 (ISBN 1-56238-5747, Pennsylvania, USA, 2005).

4. Clinical and Laboratory Standards Institute (CLSI), Estimation of total analytical error for clinical laboratory methods, (CLSI EP21-A, 2003).

5. Menditto A, Patriarca M, Magnusson B. Understanding the meaning of accuracy, trueness and precision. Accreditation Quality Assurance 2007; 12(1): 45-47. Available from: https://www.infona.pl/resource/ bwmeta1.element.springer-19cf153a-7814-327e-a001463c52edfcaa [Google Scholar].

6. Clinical and Laboratory Standards Institute (CLSI), Evaluation of the linearity of quantitative measurement approved guideline (2nd Ed.), (CLSI document EP6-A, ISBN 1-56238-498-8, Pennsylvania, USA, 2005).

7. International Organization for Standardization (ISO), General requirements for the competence of testing and calibration of laboratories, (ISO/IEC 17025, 2005).

8. WHO. Laboratory Quality standards and their implementation. WHO 2011. Available from https:// apps.who.int/medicinedocs/documents/s22409en/ s22409en.pdf?ua=1

9. Westgard JO, Carey RN, Wold S. Criteria for judging precision and accuracy in method development and evaluation. Clin Chem 1974; 20(7): 825-833. [PubMed/ Google Scholar].

10. Harr KE, Flatlend B, Nabity M, Freeman K. ASVCP guidelines: allowable total error guidelines for biochemistry. Vet Clin Pathol 2013; 42(4): 424-436. [PubMed/ Google Scholar].

11. Bland JM, Altman DG. Statistical methods for assessing agreement between two methods of clinical measurement. International Journal of Nursing Studies 2010; 47(8): 931-936. Available from: https:// www.sciencedirect.com/science/article/abs/pii/ S0020748909003204 [https://doi.org/10.1016/j. ijnurstu.2009.10.001/ Google Scholar]. 